\title{
Analyse narrative d'une dynamique d'écroulement : la Volkspolizei face aux manifestations de Leipzig, 9 octobre 1989 (partie 1)
}

Fabien Jobard

\section{CpenEdition}

Journals

Édition électronique

URL : http://journals.openedition.org/conflits/959

DOI : $10.4000 /$ conflits.959

ISSN : $1777-5345$

Éditeur :

CCLS - Centre d'études sur les conflits lilberté et sécurité, L'Harmattan

Édition imprimée

Date de publication : 1 septembre 2003

Pagination : 43-82

ISBN : 2-7475-5377-9

ISSN : 1157-996X

Référence électronique

Fabien Jobard, « Analyse narrative d'une dynamique d'écroulement : la Volkspolizei face aux

manifestations de Leipzig, 9 octobre 1989 (partie 1) », Cultures \& Conflits [En ligne], 51 | automne 2003, mis en ligne le 04 février 2004, consulté le 30 mars 2021. URL : http://journals.openedition.org/ conflits/959; DOI : https://doi.org/10.4000/conflits.959

Ce document a été généré automatiquement le 30 mars 2021

Creative Commons License 


\title{
Analyse narrative d'une dynamique d'écroulement : la Volkspolizei face aux manifestations de Leipzig, 9 octobre 1989 (partie 1)
}

\author{
Fabien Jobard
}

Une cité(...)où la paix est un effet de l'inertie des sujets conduits comme un troupeau et formés uniquement à la servitude mérite uniquement le nom de solitude plutôt que celui de Cité.

Spinoza, Traité politique, V, 4

L'écroulement de la RDA se rappelle à nous par trois images : la chute du Mur de Berlin bien sûr, au soir du 9 novembre 1989; mais aussi ces manifestations régulières, silencieuses et bientôt massives, à Dresde et Leipzig, durant l'automne 1989; ces groupes ou familles, enfin, massées aux abords des ambassades de RFA, à Prague ou Budapest, dès l'été $1989^{1}$. Deux traits pesaient fortement sur ce qui précéda la chute du Mur: le caractère d'abord illégal de l'action collective, autrement dit une prise de risque considérable de la part des participants; et, de ce fait même, une grande indétermination des répertoires d'action, qui s'étendaient de l'émigration à la participation à des manifestations de rue. Cette pluralité des formes d'action interrogeait même la notion d'action collective : comment comprendre la décision individuelle (ou familiale) d'émigration? Acte de protestation, ou acte de fuite?

Les premières analyses se sont ainsi d'emblée rangées sous la tutelle rassurante du binôme voice / exit ${ }^{2}$. Lui seul semblait propre à relever le défi que Hirschman résumait en ces termes: "Le vrai mystère des événements de 1989 est la transformation de ce qui s'engagea et fut voulu comme une activité purement privée- l'énergie déployée par des individus effrayés pour déménager d'est en ouest - en un large mouvement de protestation publique ». Distinguer ainsi les répertoires "privés » et "publics " d'action pose un risque évident d'anachronique substantialisation des concepts d'analyse: était-il finalement aussi "privé » que cela de rejoindre les rangs des candidats à l'émigration au travers des trous du rideau de fer? N'était-il pas évident qu'attendre en gare de 
Dresde, avec plusieurs milliers d'autres, le passage du train vers la Hongrie et la Tchécoslovaquie constituait un acte de défi collectif à l'encontre du régime, plus valeureux encore que l'acte manifestant lui-même, placé sous le regard et la surveillance des journalistes étrangers? Ainsi, la qualification même de ce qui se jouait alors impose une thick descriptiondes événements eux-mêmes : l'analyse narrative de ces journées peut seule rendre compte des basculements de sens, des motivations à la mobilisation et des configurations circonstancielles qui naissent et se recomposent au cours même de l'action. Nous privilégierons ici, donc, une analyse narrative (ou narration sociologique) d'un "événement-clef », la journée du 9 octobre 1989 à Leipzig, où, à la sortie du prêche de la Nikolaikirche, policiers et «manifestants" se firent face ${ }^{4}$. Ce 9 octobre signa l'écroulement irrémédiable du régime.

"Analyse narrative »: ironie de l'histoire (des sciences sociales), c'est aussi en 1989, au moment du Bicentenaire de la Révolution française et de l'activité autour de cette période, que les travaux empiriques se multipliaient, qui répondaient aux exigences épistémologiques appelant depuis une vingtaine d'années à une redécouverte exigeante de l'événement ${ }^{5}$. Episodes et journées révolutionnaires livraient, dans ces nouvelles manières de voir la Révolution, une analyse de l'événement abandonnant à la fois la simple restitution documentée des faits bruts et l'analyse de leurs causes plus ou moins lointaines, pour privilégier ce qui se jouait vraiment dans la participation de foules anonymes à des épisodes d'une brutalité parfois inouïe. Gestes individuels héroïques, désordres anomiques mus par la faim ou la peur, ou bien mobilisation collective en vue d'un basculement politique ? L'événement ne devenait plus seulement récit de ce qui se fait, mais explication de ce qui se voit, se dit et s'échange dans les vicissitudes mêmes

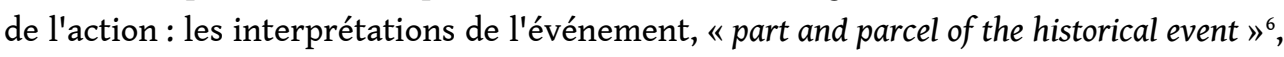
en changent, dans son cours même, à la fois le sens, mais aussi le devenir. L'analyse narrative aspire ici à s'ouvrir à l'analyse événementielle ${ }^{7}$, et en même temps à l'examen des dimensions cognitives de l'action collective ${ }^{8}$ en jeu au cours de l'événement.

Centrant l'analyse sur une journée singulière, celle du 9 octobre 1989, à un moment où à la fois, nous le verrons, tout était possible et où rien n'était encore bien défini (ni leader, ni champ de la protestation déjà là̀), nous voulons saisir pourquoi cette journée fit s'écrouler le régime de RDA ; "pourquoi ? ", autrement dit « comment? ». Comment ce «rendez-vous» fut préparé; ce à quoi les parties en présence s'attendaient; comment il se déroula et comment il fut vécu; ce qui en résulta.

Retournons au caractère par définition illégal de l'action protestataire collective: décisif, en ce sens, sera l'examen de l'anticipation des risques, et notamment des risques de répression, par le régime. Toutefois, comprendre ces évaluations des risques lors d'événements aussi fluides et incertains que ces journées, où tout, on le verra, semblait à chaque instant possible, ne peut faire l'économie d'une analyse croisée des anticipations, qui prennent en compte non seulement les cadres d'interprétation des protestataires, mais aussi les cadres de perception et les répertoires d'action disponibles dans le camp d'en face, dans les rangs des forces répressives ${ }^{10}$.

Cette problématique impose donc d'abord l'examen du maintien de l'ordre en dictature : qu'est-ce que «maintenir l'ordre » dans un État policier ? La « surprise » des événements de 1989 a consisté à voir la police ne pas réagir « comme prévu ». Mais saiton ce qui, au juste, était « prévu » en cas de mobilisation collective, dans l'Allemagne de l'Est des années 1980 ? A-t-on seulement prévu quelque mesure policière que ce soit, au sein de cette société si policée? Nous verrons alors que le maintien de l'ordre était un 
métier presque inconnu en RDA, notamment au regard du fait que la " police d'ordre " ne disposait, dans cette société si policée, d'aucune sorte d'autonomie.

Indifférenciation de la force, désectorisation de la police d'ordre : ce sont ces deux éléments qui pesèrent sur les coups joués dans le cadre des actions collectives de 1989. Ils nous permettent de mieux comprendre l'écroulement survenu à l'automne, de construire une sociologie de l'effritement de la crainte et du délitement de la contrainte.

Car lorsque la force est partout et la violence nulle part, le surgissement d'interactions de face à face entre les acteurs policiers et la population se cristallise sur une seule question: la probabilité de la violence physique. Or, la dynamique de la situation en rend soudain l'occurrence peu probable: le jeu tendu rend en quelque sorte automatique le gel de l'emploi de la force et la débandade bien ordonnée de la police.

Ce qui se joue alors, c'est une dynamique propre à cette situation d'écroulement, qui voit l'institution policière forger sur le lieu même de sa retraite une pleine autonomie, et le champ politique subir de plein fouet cette soudaine inversion hiérarchique pour se retirer naturellement d'un jeu qu'il ne maîtrise plus.

Ces épisodes offrent ainsi matière à une réflexion visant dans un même mouvement la notion d'événement, l'analyse des mobilisations, et la sociologie des changements de régime politique.

Maintenir l'ordre en dictature : retour sur un dispositif vacant

S'il est question de manifestation en RDA, une image vient en mémoire : la répression par les chars soviétiques, le 17 juin 1953 à Berlin Est, du soulèvement ouvrier et étudiant : la disproportion des moyens employés et le degré de violence physique (on compte environ 200 morts au cours de ces affrontements) illustrent alors la force du parti unique, confondue avec la supériorité militaire de l'Etat dans les épreuves de rue. L'image est trompeuse. Car elle est la seule: de ce 17 juin 1953 à novembre $1987^{11}$, presque aucune démonstration publique n'est venue troubler le cours tranquille du socialisme réel en RDA, si l'on excepte les tensions liées aux rencontres du championnat national de football (et le brusque déploiement de bannières et étendards par des Verts ouest-allemands, sur Alexanderplatz le 12 mai 1983, de $11 \mathrm{~h} 50$ à... 11h55 ${ }^{12}$ ). Les raisons de cette absence renvoient bien entendu au degré extrêmement élevé de prise de risque des manifestations publiques et/ou collectives d'hostilité politique. La rue, dans cet ordre d'idées, se trouvait donc d'autant plus déserte d'investissements protestataires. D'autres canaux, bien sûr, véhiculaient les formes de contestation les plus diverses, parmi lesquels les " pauses » durant le travail en usine ${ }^{13}$ ou les Eingaben, ces courriers de protestation, adresses ou interpellations individuelles, dont les plus diverses institutions se voyaient destinataires ${ }^{14}$.

Ainsi, l'état des modes de contestation en RDA repose sur l'effacement de la rue comme lieu de la protestation collective, et sur la rareté des épreuves de face à face, la rareté, tout simplement, des manifestations, au sens d'occupations collectives et revendicatives de l'espace public en vue, entre autres, de s'assurer une certaine visibilité à l'égard de tiers (public et/ou destinataires de la protestation). Dans un tel contexte d'apathie publique, le lieu pertinent de l'ordre public n'est plus, comme en démocratie occidentale, la rue. Cette alchimie en apparence si douce, puisque sans violences apparentes, est le produit, bien sûr, d'un immense travail disciplinaire, préventif, de contrôle social par les agences de l'Etat, visant à empêcher toute protestation publique, afin, avant tout, de ne pas avoir à se livrer à l'épreuve, publique 
elle aussi, de la répression ${ }^{15}$. Et lorsque surviennent les événements troubles de 1989, c'est toute l'économie de l'apathie publique, fondée sur la rareté des épreuves de faceà-face, qui bascule. La «structure de la conjoncture $»^{16}$ est alors découverte : l'imprévu des rassemblements, la pure contingence, met à nu la structure des rapports de force anciens, en quelque sorte jamais advenus.

Périls de l'ignorance : les effets des décennies d'apathie

Qu'est-ce que le maintien de l'ordre dans une société caractérisée par une minimisation absolue des prises de risque dans l'espace public? Insistons sur un point, celui de l'occurrence de la force physique dans les opérations de gestion des foules. Durant son audition devant la commission d'enquête sur les violences policières de Berlin durant les 7 et 8 octobre 1989, le général Streletz, membre du ministère de la défense nationale et du Conseil de défense nationale, qui gérait toutes les questions militaires et de sécurité intérieure, déclarait: "Je peux en outre ajouter qu'au moins depuis que je suis secrétaire du Conseil de défense nationale, c'est-à-dire depuis février 1972, le Conseil de défense nationale ne s'est jamais consacré qu'à des questions touchant à la défense du territoire de la RDA, et il ne s'est jamais présenté un seul cas où au Conseil de défense nationale il s'est agi de discuter ou de décider sur des questions de sécurité intérieure. Et ceci peut se vérifier dans toute une série complète de documents ".

Tout ceci pose un problème commun aux policiers et aux sociologues : ces derniers ne peuvent bien entendu dégager un nombre suffisant d'événements pour tenter de comprendre les logiques policières en action; les premiers non plus. Première conséquence : l'usage de la force dans des interactions avec des groupes protestataires provient bien plus des effets de la méconnaissance et des erreurs de calcul que d'une volonté politique ou policière bien déterminée. Un bon exemple nous est offert par le concert putatif des Rolling Stonesà Berlin Est en 1969 ${ }^{17}$. Du fait de la rareté de l'information et l'étanchéité à l'égard du monde occidental, les rumeurs tenaient souvent lieu d'information (au point que, comme on le verra en 1989, un des instruments clefs de la maitrise de la situation par le gouvernement est l'initiative et le contrôle de la rumeur). En septembre 1969, la radio ouest berlinoise annonce que les Rolling Stonesdonneront un concert au soir du 7 octobre (jour anniversaire des vingt ans de la République) sur le toit du bâtiment des éditions Springer, qui du côté Ouest de la ville surplombe le Mur. Quel qu'ait été le degré de croyance en cette information, des milliers de jeunes se pressent à l'endroit pressenti dès l'après-midi. La Volkspolizeiréagit dans la plus grande panique et emploie massivement charges, matraquages et interpellations pour faire cesser ce que, face au déficit de connaissance et de savoirfaire pratique de ce qu'est un rassemblement "spontané », elle voit comme une atteinte fondamentale à l'ordre et la sécurité. Un autre événement de ce type se produisit en 1987, lorsque différents groupes musicaux ouest-allemands, quelques jours avant la visite de Ronald Reagan, donnèrent un concert au pied du Mur ${ }^{18}$.

Trois points sont donc à retenir. Le premier est la carence, pratique et théorique, en matière d'apprentissage des modes de maintien de l'ordre de la part de la police ordinaire en RDA. On le sait, l'efficacité du maintien de l'ordre repose sur l'enregistrement systématique par la police des événements passés, notamment de ceux qui ont fait l'objet d'altercations violentes. La police dispose dans ces archives d'une mémoire des événements protestataires, qui permet de fonder les répertoires d'action et les dispositifs d'intervention qui seront employés lorsque des agriculteurs, des étudiants ou des petits commerçants, pour citer les groupes qui témoignent d'une 
fréquence accrue du recours à la violence, viennent à se mobiliser. D'une manière bien moins systématique, et surtout plus récente, la police ouest-allemande a également commencé à constituer une mémoire institutionnelle des activités protestataires de manière à limiter la prise de risque sur le terrain et le recours à la force ${ }^{19}$. Le succès des technologies d'évitement de la force et de l'interaction de face-à-face, ainsi que des technologies de mise à distance des foules, repose sur une connaissance approfondie des répertoires supposés des groupes adverses grâce à la mémoire archivée des mobilisations passées ${ }^{20}$.

Dans les démocraties ouest-européennes, la défaillance des outils d'anticipation des comportements des groupes adverses est l'une des sources majeures d'occurrence de la violence : la rationalisation de l'emploi de la force dans le cours de l'action repose sur la rationalité des outils de prévision et de calcul. Point étonnant de constater que les deux manifestations est-berlinoises que nous avons évoquées, par leur caractère "spontané ", fussent les deux seules qui, avant 1989, aient vu se dérouler des violences; de la même manière que les infirmières, groupe n'ayant jamais manifesté, formé de personnes peu familières des usages manifestants, défilant en 1988 aux abords du Palais de l'élysée pour gagner contre le Premier ministre Michel Rocard le soutien du Président, appellent une réponse disproportionnée de la part des forces de l'ordre (emploi des canons à eau, notamment, qui depuis cet événement, n'ont jamais été ressortis qu'à l'occasion de la répression de la manifestation lors du débat parlementaire sur les retraites à l'été 2003).

Le maintien de l'ordre : le maillon faible

Le deuxième point à retenir est la sous-professionnalisation des forces de maintien de l'ordre en RDA. Le signe le plus tangible est l'absence de formation en la matière, ainsi que le sous-équipement en moyens de protection individuels et collectifs. La Volkspolizeiprésente en effet le visage d'une institution à rebours de toutes les politiques de rationalisation de la compétence professionnelle dans le champ policier promues, parfois depuis plus d'un siècle, dans les démocraties occidentales ${ }^{21}$. Ces carences sont d'autant plus marquées selon la technicité des missions de police, à commencer par l'usage des véhicules, des machines à écrire et des moyens de communication-radio, trois outils pour le moins fondamentaux du métier policier. En ce qui concerne les véhicules, les policiers de Berlin Ouest constateront sans surprise que les fameuses Trabant, au mieux les Wartburg, qui équipent la Volkspolizei, affichent un résultat aux tests de résistance aux chocs à peu près équivalent à ceux des véhicules de l'Ouest des années 1950. Pire encore, une note portée à la connaissance de la police de Berlin Ouest le 18 septembre révélait par exemple que seuls $40 \%$ des Vopos sont en possession d'un permis de conduire ${ }^{22}$. L'usage impropre, lent et maladroit de la machine à écrire se verra d'ailleurs après la réunification décrié par les chefs de poste de l'Ouest qui accueillent des anciens Vopos.

On retrouve cette opposition, de manière plus surprenante, en ce qui concerne les équipements, notamment en moyens armés. Ce n'est qu'en 1960 que la direction centrale de la Volkspolizei a décidé l'uniformisation des armes individuelles, constituées jusque lors du rassemblement désordonné d'une quinzaine de modèles différents, empruntés à l'ancienne armée allemande ou à l'armée soviétique : le pistolet soviétique Makharov $9 \mathrm{~mm}$ est ainsi devenu l'arme de poing de tous les policiers de sécurité publique. En 1968-69, les policiers ont eux aussi été pourvus de moyens spécifiques en matière de maintien de l'ordre, soit un petit fusil mitrailleur et des boucliers de 
protection en plastique. Les forces d'intervention spéciales se voyaient, de leur côté, équipées de Makharov, de fusils, de fusils-mitrailleurs Kalachnikov et d'équipements antichars. La même année, les Vopos (tous comme leurs collègues de Berlin ouest) ont été en outre équipés de nouveaux uniformes, plus légers et des casquettes plus souples qui ont aboli le Tschako, le fameux casque des policiers est- et ouest-berlinois qui, en $\mathrm{RDA}$, équipait tous les policiers du territoire ${ }^{23}$. Il semble que ces équipements soient restés inchangés, en ce qui concerne les policiers de sécurité publique et de police judiciaire, jusqu'à la fin des années 1980, même si un rapport remis au ministère de l'Intérieur en 1984 mentionne l'introduction de pistolets Skorpio de calibre de 7,65 $\mathrm{mm}$ : la dégradation sensible du budget de l'État dans les années 1980 n'a sans doute pas permis un renouvellement notable de ces équipements et armements qui, donc, dans le meilleur des cas, avaient été introduits au cours des années $1970^{24}$. Une note confidentielle du ministère de l'Intérieur de 1983 indiquait que la durée maximale d'emploi des technologies, équipements aussi bien que des bâtiments, moyens de transport etc. devait être prolongée le plus longtemps possible, par toutes les mesures appropriées en matière d'entretien, de remise en état et $\mathrm{d}^{\prime} \mathrm{emploi}^{25}$ : ce qui signifie que l'économie de pénurie touchait le cœur de l'exercice de la puissance de l'État-Parti.

Et cette sous-professionnalisation se transporte de l'état des matériels à l'incurie des entraînements. Aussi surprenant que cela puisse paraître, aucune mesure spécifique n'était prévue en cas d'échec de la dissuasion préalable des rassemblements publics. Pour le dire autrement, aucun dispositif n'était prévu en cas de... manifestation. Prenons en pour témoins les mesures prises au sein des groupes «civils » de combat (les «Kampfgruppen", les milices de travailleurs armés) face aux premiers signes tangibles d'agitation au printemps $1989^{26}$. Ces milices avaient été mises sur pied après les événements de juin 1953 afin de disposer de forces armées aptes à réprimer les éventuelles protestations publiques au sein des usines, avant précisément qu'elles ne gagnent la rue. Le ministère de l'Intérieur (MfS) était responsable de leur équipement et de leur entraînement. Au printemps 1989, ces groupes furent destinataires d'un plan d'entraînement secret (sitôt révélé dans la presse ouest-allemande ${ }^{27}$ ) visant à la formation aux combats de rue. Ainsi, à Leipzig, les entraînements débutèrent en avril 1989, qui visaient à répondre au cas où « des cercles évangéliques exalteraient la population et où l'on en viendrait à des exactions ». La nouveauté de ce plan, qui visait l'emploi de matraques et de boucliers de protection, était telle que des membres de ces milices protestèrent: "De jeunes miliciens se sont exprimés sans relâche tout autour d'eux, disant qu'ils ne s'étaient pas engagés dans les milices pour ça. Les milices n'ont pas été fondées, selon eux, pour être des 'gardes de matraqueurs' et ils se refusent à 'frapper des tiers' » (rapport du 7 avril 1989 adressé à la direction locale de la $\mathrm{MfS}^{28}$ ).

Le veto opposé au recours à la force s'explique, bien sûr, par les signes de vacillement de l'autorité du Parti qui, çà et là, et notamment en Saxe, se multiplient déjà à cette époque; ces signes d'incertitude qui avaient précisément conduit la direction des Kampfgruppenà introduire ces nouvelles modalités d'emploi de leurs troupes. Mais ce n'est pas tant l'opposition ponctuelle des troupes à leur hiérarchie qui retient ici l'attention, que l'objet même de l'opposition : cette décision, prise en 1989, d'équiper et de former des groupes de combat en vue... du combat. Il faut souligner ce point: ramener ces marques soudaines de désobéissance dans les rangs des Kampfgruppenà un strict «effet de conjoncture » (l'air du temps en ce printemps 1989) ne permet pas de comprendre l'importance de la rupture induite par le caractère même de l'ordre donné, en l'occurrence se former à l'exercice de la force contre des concitoyens. «L'air du 
temps » accentuait la probabilité d'un refus d'obéissance. Mais ce dernier se cristallisait sur la question de l'emploi effectif de la force, preuve du caractère nodal de cette modalité jamais advenue, depuis 1953, de l'exercice de la domination du Parti unique en $\mathrm{RDA}^{29}$.

\section{Police et politique}

Un troisième point émerge comme conséquence partielle du premier. Plus encore que dans les démocraties occidentales, où de tous les métiers policiers les liens entre la "police d'ordre » et l'Exécutif sont des plus ténus, la police est-allemande se trouve en matière de maintien de l'ordre dans une situation de sujétion et de dépendance totale à l'égard du pouvoir. Point besoin de revenir sur la sujétion : c'est là un trait commun en matière de maintien de l'ordre que de réduire au maximum la distance qui sépare autorité politique et instrument policier ${ }^{30}$. Le trait spécifique de la police d'ordre en RDA est son sous-développement technique et donc l'indispensable nécessité de la solidité des dispositifs préventifs. Ceux-ci ne sont bien entendu pas seulement policiers : ils mettent en œuvre différents acteurs guidés avant tout par les agents de renseignement du Parti. Aussi, durant même les opérations de maintien de l'ordre, le travail policier se trouve minimisé au profit d'un travail de présence accrue des forces de la Stasi, d'interventions ciblées de celles-ci auprès des personnes-cibles, en bref : une réduction maximale de l'autonomie de décision et d'action de la police. Ainsi, lors de la manifestation à Berlin de quelques dizaines d'oppositionnels protestants, le jour anniversaire de l'assassinat de Rosa Luxembourg et Karl Liebknecht, le 9 janvier 1988, quelques forces de police sont déployées pour assurer le bon ordre du défilé des personnalités officielles au monument aux morts, mais ce sont 300 " forces sociales " (soit, «collaborateurs informels » de la Stasi), en compagnie "d'agents de la Stasi de l'arrondissement de Berlin » et de " camarades responsables du SED berlinois » qui se chargent du repérage et de la mise à l'écart des manifestants ${ }^{31}$. La police, ce jour-là, n'intervient pas.

Le dispositif de maintien de l'ordre public en RDA est alors caractérisé par: le surinvestissement dans la dissuasion des rassemblements publics; le sousdéveloppement des techniques d'emploi et de maîtrise de la force, et, accessoirement, une dépendance totale de l'instrument policier au pouvoir politique (traduction de l'exiguïté de sa marge de manœuvre technique). Tous ces éléments dessinent la trame organisationnell ${ }^{32}$ de ce qui se jouera au 9 octobre, et qui éclatera sans retour possible, sous l'effet de l'effacement des frontières établies entre les différents secteurs de la société (à commencer par la partition police/pouvoir).

Mesure de l'efficacité des stratégies d'escalade : la peur

Dans son court article sur les mobilisations en Europe de l'Est en 1989, Michel Dobry va un peu vite en besogne lorsqu'il explique qu'il y a eu, en Allemagne de l'Est ou en Tchécoslovaquie une " renonciation ostentatoire et rapide à l'usage de la force " ${ }^{33}$. En réalité, les premières mobilisations d'importance qui ont déterminé le sort de la RDA se sont déroulées dès novembre 1988 à Berlin, dans le quartier de Prenzlauer Berg. On peut les retenir comme étant d'importance en ce qu'elles ont effectivement dépassé le seuil probant constitué par le fait que les gens rassemblés ne se connaissaient pas déjà tous de vue ; par le fait, donc, que la mobilisation des deux ou trois centaines de personnes autour de telle ou telle église dépassait le cercle des connaissances familières. A ce moment-là, vu le caractère proprement inédit de l'émergence de manifestations 
publiques, la question de l'emploi de la force s'imposait à tous comme la question nodale de la confrontation politique entre le SED et les protestataires.

Cycles exceptionnels et cycles ordinaires : le dérèglement de l'horlogerie politique

Côté protestataires, s'offraient des opportunités en vue de micro-tests destinés à préciser les positions de l'adversaire. Côté policier, Erich Mielke lui-même, chef du MfS et de la Stasi, reconnaissait en octobre 1988 un resserrement indéniable des marges de manœuvre répressives du fait de l'environnement international, encadré à l'Est par une "loyauté " incertaine de la part du Grand frère soviétique et à l'Ouest par une intégration croissante dans les marchandages financiers avec la RFA et l'OCDE : « Nous ne pouvons sous aucun prétexte offrir à l'adversaire les munitions dont il pourrait s'emparer pour qualifier nos organes [répressifs] de fauteurs de troubles au sein du processus de détente et de paix $»^{34}$.

Les contestataires constatent ainsi dès janvier un élargissement de l'espace de la protestation, mais qui ne permet pas à leurs yeux une prise de risque trop grande : on reste le plus souvent dans les églises ou sur la petite place qui les entoure ${ }^{35}$, on y chante des chansons interdites, on y constitue des bibliothèques clandestines, on y tient des concerts de rock... La peur se mesure donc d'abord à la retenue de la portée des coups joués. Elle se matérialise également par les signaux de plus en plus fréquents qu'adresse la direction du Parti aux manifestants potentiels. La répression des mobilisations en Chine le 4 juin 1989 fournit une ressource de premier choix pour le gouvernement qui, à l'aide d'alertes lancées dans la presse officielle, exerce la menace permanente du recours à ce que l'on appelle alors " la solution chinoise ${ }^{36}$ ». Le gouvernement exploite sans retenue ces "prophéties du malheur $\rrbracket^{37}$ en n'hésitant pas à envoyer à Pékin une délégation officielle formée notamment de Egon Krenz et du secrétaire général du SED berlinois, Günter Schabowki. La menace est d'ailleurs d'autant plus crédible que, les seuils de mobilisation ordinaires ayant tous été dépassés par la fréquence et l'ampleur des mobilisations, le gouvernement peut s'autoriser une situation de jeu tendu pour mettre en œuvre une solution chinoise dont il sait qu'il souffrira également des conséquences, non seulement par rapport à Moscou, mais aussi par rapport aux nouvelles conditions d'emploi de la force armée à des fins intérieures depuis la CSCE d'Helsinki ${ }^{38}$.

En réalité, un foyer indéniable de tension résidait dans la tenue prochaine des élections municipales, prévues pour le 7 mai 1989. La présentation des candidats des partis officiels fut émaillée, notamment à Berlin Est, d'interruptions de séances provoquées par des contestataires souhaitant inscrire telle ou telle question à la liste des débats publics, notamment la question de l'environnement, voire pour proposer leurs propres candidats. Pour la première fois en avril 1989, les forces de police intervinrent publiquement pour faire cesser les troubles constatés dans une de ces réunions ${ }^{39}$.

Et à partir du 7 mai 1989, à Berlin, jour des élections communales, un cycle de mobilisation se met en place qui a pour effet d'introduire, dans la temporalité centralisée et parfaitement ordonnée du gouvernement est-allemand (rythme politique hebdomadaire marqué par la réunion chaque mardi du Comité central; rythme annuel marqué par le jour-anniversaire du 7 octobre; différents rythmes institutionnels : le 1er juillet est par exemple le "Tag der Volkspolizei ", marqué par des réjouissances internes), une autre temporalité, celle de la contestation ${ }^{40}$. Pour marquer leur indignation face au truquage du résultat des élections, une mobilisation mensuelle se tient dans les rues de Berlin, tous les sept du mois. L'engagement de ce cycle fut facilité, 
précisément, par le resserrement des marges de manœuvre répressives éprouvé par le gouvernement : événement public, le scrutin communal ne pouvait se tenir à huis clos, non plus sans la présence de la presse étrangère, qui réduit ainsi à néant tout espoir d'intervention publique des forces répressives et augmentait terriblement le coût des interventions "préventives" (interpellations aux domiciles ou dans les transports publics, notamment). Les lieux d'exercice du vote, notamment, se trouvaient ainsi neutralisés; et les contestataires purent ainsi disposer à Berlin, dans 66 des 67 bureaux de vote, d'observateurs indépendants ${ }^{41}$. La fraude électorale n'en fut que moins contestable; l'indignation ultérieure que plus grande. Le cycle de protestation épouse donc les vicissitudes des cycles politiques officiels. L'été passé, et tandis que les contestataires berlinois manifestent leur désapprobation publique chaque $7 \mathrm{du}$ mois, ce sont désormais les préparatifs du Quarantenaire de la république démocratique prévu en octobre qui multiplient les foyers de tension, le plus souvent autour des églises. Le lundi 11 septembre, le prêche de l'église Nikolai à Leipzig offre au gouvernement l'occasion de tester l'option répressive par l'arrestation de 89 participants à la sortie de l'église. Tous seront condamnés, dont 19 à plusieurs mois de prison ferme.

S'ouvre alors un cycle de mobilisation cette fois hebdomadaire, après le sermon prononcé à l'église, appelant tous les lundis, à la libération des manifestants incarcérés à Leipzigi2. L'émergence de cette temporalité fracture la fixation centralisée des rythmes politiques et représente de ce fait une menace considérable à l'égard du gouvernement qui, par ailleurs, voit son propre environnement marqué par une incertitude croissante, due au rétablissement de la liberté de circulation en Hongrie à partir de l'été, qui amène des dizaines de milliers de ressortissants de la RDA à fuir le territoire national. Cette situation d'incertitude se cristallise à Berlin, lorsque se confrontent le calendrier officiel (fête des 40 ans de la RDA prévue le 7 octobre, marquée par la présence de Gorbatchev à Berlin) et le calendrier protestataire : les 6, 7 et 8 octobre, se produisent à Berlin deux jours et deux nuits de répression policière à l'encontre des manifestants. Cette répression fera d'un coup monter la tension à Leipzig lorsque le lundi 9 octobre les manifestants se réunissent autour de l'église. Aux yeux de tous, il est clair que le gouvernement ne peut faire l'économie d'une nouvelle défaite dans l'ordre du maintien de l'ordre public.

Leipzig : le lieu de l'épreuve finale

La manifestation de Leipzig constitue le moment d'inflexion définitive du régime. La raison en est la force de la peur, devenue à Leipzig ${ }^{43}$ seul «signe du lien » entre policiers d'un côté et manifestants de l'autre, donc seul signe du lien qui unit le Parti aux forces protestataires. Le signe du lien est, chez Goffman, l'ensemble des modes de qualification de la nature de la relation qui unit deux acteurs ${ }^{44}$. Ils sont ces idiomes qui permettent à deux personnes qui se rencontrent pour la première fois de témoigner réciproquement qu'ils ne veulent pas engager de relation ou au contraire qu'ils souhaitent « ancrer » la relation. L'intérêt de cette notion est ici double. Elle permet de qualifier la nature de la confrontation entre policiers et manifestants, et de comprendre ce qui, dans le cours de la confrontation, permet aux protagonistes de disposer d'éléments de définition de la situation. Elle permet également de bien prendre conscience de la réduction de tout le conflit, dans ses dimensions politiques et policières, à l'intensité de la peur, et donc à sa seule dimension policière.

Les manifestations de Leipzig consistaient depuis septembre à occuper la rue, sans donner de contenu nécessairement très clair à cette occupation ${ }^{45}$. Ces rassemblements 
constituent de la même manière qu'à Berlin des tests de position à l'égard du pouvoir central. La superposition des calendriers, dont nous avons parlé, produit le 9 octobre sa propre logique : chacun sait que, dans le contexte des répressions de Berlin, Leipzig ce 9 octobre sera le lieu d'une véritable expérimentation politique. De son côté, le gouvernement accroît la densité des alertes, en mettant en scène dans la ville, les jours qui précèdent, un décor de préparation à la guerre civile et en publiant dans le journal local Die Leipziger Volkszeitung, pour la première fois, la lettre du chef de l'un des Kampfgruppen ${ }^{46}$ locaux selon lequel les armes à feu seront employées ${ }^{47}$.

Pourtant, hors des scènes officielles ${ }^{48}$, les consignes se diluaient dans un flou généralisé. La résolution du chef de la Volkspolizeide Leipzig, en charge de la coordination du maintien de l'ordre, fait part le 6 octobre des dispositions écrites suivantes au ministre de l'Intérieur et chef de la Deutsche Volkspolizei, le général Dickel :

État de la situation

Dans les dernières semaines, le nombre des participants aux "prières du lundi » s'est considérablement élevé (de 800 à 8000). L'agressivité à l'égard des organes de protection et de sécurité, en particulier à l'égard de la Deutsche Volkspolizei, a clairement augmenté. Le 9 octobre 1989, il est attendu un nombre de manifestants d'à peu près 50000 personnes. Cette estimation se fonde sur la tenue de la foire annuelle, qui amène un grand nombre de déplacements individuels, une situation explosive, à laquelle s'ajoute une agressivité croissante et le fait que de la part des représentants de l'église, aucun appel au calme n'émerge, mais c'est bien plutôt le désordre qui est encouragé. (...)

Camarade ministre!

En vertu des ordres reçus de vous et de la situation ainsi exposée, j'ai décidé (...) :

1.- de garantir la stabilité des manifestations tenues pour le 40ème anniversaire de la $\operatorname{RDA}(. .$.$) ;$

- dans le centre de la ville, en particulier, d'assurer l'ordre et la sécurité publics ;

- d'interdire les désordres à prévoir autour de l'église Nicolas, de les identifier dès qu'ils se produisent et de lutter sans réserve contre les provocations et autres tentatives de désordre (...) ;

3.- d'employer tous les moyens et les forces pour former des troupes d'intervention à partir des éléments suivants: enquêteurs, forces de sécurisation, de contrôle, de circulation publique, de dissolution et d'interpellation, forces spéciales, forces de réserve ;

La suite 


\section{NOTES}

1. Ce texte est la version légèrement remaniée du Working Paper $n^{\circ} 14$ publié par le Centre Marc Bloch de Berlin, auquel s'adressent mes remerciements pour permettre la présente publication.

2. . Voir au sixième jour qui suivit la chute du Mur l'analyse proposée par Henning Ritter, directeur de la section des sciences sociales et humaines de la Frankfurter Allgemeine Zeitung dans les colonnes de son journal : « Abwandern, Widersprechen. Zur aktuellen Bedeutung einer Theorie von A.O. Hirschman ». Et, dans le champ scientifique, la contribution de Detlef Pollack, sociologue et théologue de RDA, et témoin des événements de Leipzig à l'automne 1989, « Das Ende einer Organisationsgesellschaft », Zeitschrift für Soziologie, 19, août 1990. Albert Hirschman revient sur ses propres cadres d'interprétation dans « Exit, voice, and the fate of the German Democratic Republic. An essay in conceptual history », World Politics, 45, janvier 1993, pp. 173-202 et de manière plus synthétique dans Un certain penchant à l'autosubversion, Paris, Fayard, 1995. Cette analyse fut également privilégiée en France : Dobry M., « Les causalités du probable et de l'improbable. Notes à propos des manifestations en Europe centrale et orientale », Cultures \& Conflits, 17, 1995, pp. 111-136. Voir toutefois les analyses en termes d'entreprises de mouvement social et l'approche critique du travail préalable à l'action protestataire par les « mouvements de citoyens » (Chibret R.-P., « Action collective et changement politique en Allemagne de l'Est. Le 'tournant' de la RDA (1989-90) », Revue française de science politique, 5, 45, oct. 1995, pp. 791-822).

3. . Hirschman A., "Exit, voice, and the fate of the German Democratic Republic », op. cit., 1993, p. 198 (souligné dans le texte original).

4. . Nous accepterons ici la définition suivante d'un événement-clef : « les événementsclefs (focal events) [sont ceux qui] portent les anticipations sociales à des niveaux extrêmes d'incertitude institutionnelle ", (Schedler A., " Taking uncertainty seriously : The blurred boundaries of democratic transition and consolidation ", Democratization, 8 (4), hiver 2001, pp. 1-22). Voir de manière plus générale sur les « focal events » ou " focal points », Thomas Schelling, The Strategy of Conflict, Cambridge, Harvard University Press, 1960, pp. 53-80 et 111-113.

5. . Nora P., « Le retour de l'événement », in Le Goff, J., Nora, P., Faire de l'histoire, Paris, Gallimard, 1974, t. 1, pp. 210-234 et Stone L., « The revival of narrative.

Reflections on a new old history ", Past and Present, 85, 1979, pp. 3-24.

6. . Sewell W., « Historical events as transformation of structures. Inventing revolution at the Bastille ", Theory and Society, 25, 1996, p. 861. Sur l'analyse événementielle comme récit simultané de l'événement et de ses modalités de lecture et d'appropriation par les acteurs eux-mêmes, voir notamment Baker K., Au tribunal de l'opinion. Essais sur l'imaginaire politique au XVIIIe siècle, Paris, Payot, 1995 (trad. de l'anglais, 1990) et Tackett T., Par la volonté du peuple. Comment les députés de 1789 sont devenus révolutionnaires, Paris, Albin Michel, 1997 (trad. de l'anglais, 1996). Voir aussi Gresle F., «Introduction à une sociologie de l'historiographie révolutionnaire. Quelques observations sur un bilan », Revue française de sociologie, XXX, 1989, pp. 371-403 (pp. 382-388 not.) et Sewell W., « Three temporalities. Toward an eventful sociology », in 
McDonald T., The Historic Turn in the Human Sciences, Ann Arbor, University of Michigan Press, 1996, pp. 245-280.

7. Ces analyses privilégient soit l'examen d'événements-clefs, soit (le plus souvent) l'examen d'une somme d'événements donnés. Voir l'excellente présentation de Tarrow S., « Studying contentious politics. From event-ful history to cycles of collective action », in Rucht D., Koopmans R., Neidhardt F. (dir.), Acts of Dissent. New Developments in the Study of Protest, Berlin, Wissenschaftszentrum/Sigma, 1998, pp. 33-64.

8. . Sur l'apport de la « labelling approach » ou « frame analysis », voir les trois contributions de David Snow, de Daniel Cefaï et de Danny Trom dans Cefaï D., Trom D., Les formes de l'action collective. Mobilisations dans des arènes publiques, Paris, Ecole des hautes études en sciences sociales, Raisons pratiques $n^{\circ} 12,2001$, pp. 9-134 notamment.

9. C'est pourquoi la narration envisagée ici ne se rapporte pas à « l'analyse narratologique » de l'école de « l'analyse des cadres » ( framing »). Voir notamment les commentaires de D. Cefaï sur les travaux de F. Poletta (« Les cadres de l'action collective ", in ibid., pp. 51-98).

10. . L'un des effets du désintérêt de la sociologie des mobilisations pour l'événement fut longtemps la cécité à l'égard des modes concrets de déroulement des manifestations, et notamment des interactions entre protestataires et forces de l'ordre. Jusqu'à ces dernières années (jusque par exemple Fillieule O., Stratégies de la rue. Les manifestations en France, Paris, Presses de la Fondation nationale des sciences politiques, 1997, pp. 54-57 ou Della Porta D., Reiter H. (dir.), The Policing of Mass Demonstrations in Contemporary Democracies, Mineapolis, University of Minnesota Press, 1998), on a surtout étudié les effets de la répression, dont l'un des paradoxes est ainsi d'encourager la délégitimation du régime en augmentant les motivations à participer : les mesures répressives isolent les victimes, qui disposent alors pour seules ressources les gratifications symboliques ou matérielles accordées par le groupe des pairs visés par la répression. Ainsi se renforcent les identités protestataires des victimes de la répression, ainsi que les socialisations alternatives, encourageant ainsi la formation de vagues croissantes de protestation (sur ces dynamiques de " protest career » et de « bridge burning », voir Marx G., « External efforts to damage or facilitate social movements. Some patterns, explanation, outcomes, and complications ", in Zald M., McCarthy J. (dir.), The Dynamics of Social Movements, Winthrop, 1979, pp. 94-129, Mc Adam D., " Micromobilization contexts and recruitment to activism », International Social Movement Research, 1, 1988, pp. 125-154 et Opp K.-D., Roehl W., « Repression, mobilization, and political protest ", Social Forces, 69, 2, décembre 1990). Les analyses de Karl-Dieter Opp sur la dynamique protestataire de l'automne 1989 en RDA s'inscrivent dans cette sociologie des effets (par exemple, «DDR'89. Zu den Ursachen einer spontanen Revolution ", in Joas, H., Kohli, M. (dir.), Zusammenbruch. Soziologische Analysen, Francfort/M, Suhrkamp, 1993, pp. 194-220).

11. . 1987 : protestations publiques contre l'interpellation de sept militants de la communauté évangélique de l'église Zionskirche (Berlin Est, Prenzlauer Berg - voir infra n. 34). Typiquement, les leaders des protestations publiques de l'année 89 , le plus souvent en effet regroupés autour d'églises évangéliques ou œcuméniques, préféraient dans les années 1980 recourir aux formes « classiques » d'expression qu'étaient par exemple les « Eingaben », lettres privées adressées à diverses autorités (cf. thèse de Jay Rowell, citée infra n. 13). Ainsi la Eingabe adressée par des « militants des droits de 
l'homme » au SED le 2 avril 1986, qui se concluait par « Nous attendons de ce fait que s'engage un dialogue constructif dans notre pays ", signée par les futurs leaders publics Bärbel Böhley, Katrin et Frank Eigenfeld, Ulrike et Gerd Poppe, Edelbert Richter, HansJochen Tschiche (citée in Süß W., Staatssicherheit am Ende. Warum es den Mächtigen nicht gelang, 1989 eine Revolution zu verhindern, Berlin, Ch.Links, 1999 (2è éd.), p. 85 n. 47).

12. . Wolle St., Die heile Welt der Diktatur. Alltag und Herrschaft in der DDR. 1971-89, Berlin, Christopher Links, 1998, p. 273.

13. . Kott S., Le communisme au quotidien. Les entreprises d'Etat dans la société estallemande, Paris, Belin, 2001.

14. . Rowell J., L'Etat totalitaire en action. Les politiques du logement en RDA 1945-1989, Paris, Université Paris 1, thèse de doctorat de science politique (dir. M. Offerlé), dactyl., 2001.

15. . Déployer tout ce que recouvre cette opposition entre public et caché (plus que public et privé), ou répressif et préventif, dépasserait naturellement le cadre de cet article. Je me permets de renvoyer, sur le fond, aux deux travaux précédents (n. 13 et 14), qui font admirablement le point sur ces questions, et, pour un champ voisin de celui du maintien de l'ordre, à Jobard F., «L'ajustement et le hiatus. La prison allemande après la Réunification ", in Lascoumes P., Artières Ph. (dir.), Gouverner et enfermer, Paris, Presses de Sciences-po, 2004 (à paraître).

16. . Sur cette expression, voir Sahlins M., Des îles dans l'histoire, Paris, Gallimard/Le Seuil, 1989 (trad. de l'anglais, 1985), p. 158. Sur les rapports de la contingence et de la structure dans le déroulement des événements, voir Sewell W., « Historical events as transformation of structures ", op. cit., 1996, p. 862.

17. . Wolle St., Die heile Welt der Diktatur, op. cit., 1998, p. 156.

18. . Voir Berlin Chronik, Munich, Chronik Verlag, 1997 (3è éd.), p. 580.

19. . Busch H., et al. (dir.), Die Polizei in der Bundesrepublik, Francfort sur le Main, New-York, Campus, 1988, pp. 353-355.

20. . Voir par exemple pour la France, Bruneteaux P., Maintenir l'ordre. Les transformations de la violence d'État en régime démocratique, Paris, Presses de la FNSP, 1996, pp. 23-26, 169-180 et 205-209.

21. . Voir Fogelson R., Big City Police, Cambridge, Harvard University Press, 1977 ; en Grande-Bretagne, Reiner R., The Politics of Police, Toronto, University of Toronto Press, 1992 (1985) ; en France, Berlière J.-M., Le monde des polices en France, Bruxelles, Complexe, 1996, pp. 69-76 ; en RFA, Busch H. et al. (dir.), Die Polizei in der Bundesrepublik, op. cit., 1988, pp. 147-169.

22. . Cellule de préparation du 3 octobre, 6ème réunion (18 septembre 1990). Ces documents sont exploités de manière plus large in Jobard F., « Usages et ruses des temps. L'unification des polices berlinoises après 1989 ", Revue française de science politique, 53, 3, juin 2003, p. 362.

23. . Pour la Volkspolizei, voir Lindenberger Th., « Die deutsche Volkspolizei (1945-90) », in Diedrich Th., Ehlert H., Wenzke R., Im Dienste der Partei. Handbuch der bewaffneten Organen der DDR, Berlin, Ch.Links, 1998, p. 134. Pour la police ouestberlinoise, voir Steinborn N., Krüger H., Die Berliner Polizei, Berlin, Arno Spitz, 1993. Sur la modernisation des polices ouest-allemandes durant les années soixante, voir Jobard F., "Les deux visages de la sécurité en Allemagne ", in Jean-Charles-Froment et al.(dir.),Les Etats à l'épreuve de la sécurité, Grenoble, Presses universitaires de Grenoble, 2003, pp. 192-216 et Weinhauer K., Schutzpolizei in der Bundesrepublik. 
Zwischen Bürgerkrieg und innerer Sicherheit, die turbulenten sechziger Jahre, Padeborn, Ferdinand Schöhning, 2003.

24. . Lindenberger Th., « Die deutsche Volkspolizei (1945-90)», op. cit., 1998, p. 134-135. 25. . « Document confidentiel présenté au Collègue du ministère de l'Intérieur », 2/83, cité in ibidem, p. 135 n. 101.

26. . Süß W., Staatssicherheit am Ende, op. cit., 1999, pp. 115-116. Sur ces milices, voir Wagner A., « Die Kampfgruppen der Arbeiterklasse », in Diedrich Th. et al., Im Dienste der Partei, op. cit., 1998, pp. 281-337 et Kott S., Le communisme au quotidien, op. cit., 2001.

27. . Spiegel, 1er mai 1989 (cité in Süß W., Staatssicherheit am Ende, op. cit., 1999, p. 115 n. 212).

28. . Ibid., p. 116 n. 214.

29. . On pourra comparer ici avec l'analyse de la désobéissance des troupes des Ateliers nationaux, à l'avant-garde des barricades de juin 1848, et de l'obéissance des troupes de la Garde nationale, en première ligne dans la répression des insurgés, in Traugott M., Armies of the Poor. Determinants of Working-Class Participation in the Parisian Insurrection of June 1848, Princeton, Princeton University Press, 1985. Traugott, réfutant l'hypothèse de Marx selon laquelle les Gardes nationaux n'étaient que des membres du Lumpen anomique, tirant sans conscience ni retenue sur leurs frères ouvriers des Ateliers, montre d'une part que l'appartenance de classe des deux groupes est sensiblement identique, mais surtout, reprenant des travaux plus anciens sur la sociologie des groupes militaires employés à la répression des insurrections, il montre l'importance décisive, quant à l'emploi de la force, des modes d'organisation et de gratification de « la base » au sein des Ateliers, ce qu'il désigne comme « hypothèse organisationnelle » contre l'hypothèse de classe.

30. . Monjardet D., Ce que fait la police. Sociologie de la force publique, Paris, La Découverte, coll. « Textes à l'appui », 1996.

31. . La note d'information de la Stasi datée du 12 janvier 1988 sur le déroulement de cette manifestation est reproduite dans « Akteneinsicht, Aktion 'Störenfried' ", in Schädlich H.-J. (dir.), Aktenkundig, Berlin, 1992, pp. 91-153.

32. . Traugott M., Armies of the Poor, op. cit., 1985. Cette structure organisationnelle, qui accueille l'événement-clef, embrasse ainsi tout l'équipement technique et juridique de l'action policière, selon des modalités que nous avons développées in Jobard F., Linhardt D., « Der Kontrolleur und Verwalter. Vergleichende Pragmatik zweier Modalitäten des 'Poliziierens' », in Allmendinger Jutta (dir.), Entstaatlichung und soziale Sicherheit. Verhandlungen des 31. Kongresses der DGS, Opladen, Leske + Budrich, 2003.

33. . Dobry M., « Les causalités du probable et de l'improbable », op. cit., 1995.

34. . Cité in Süß W., Staatssicherheit am Ende, op. cit., 1999, p. 85 n. 49.

35. . Ces églises (Zionskirche, Bethelskirche et Samariterkirche, toutes trois à Prenzlauer Berg) sont ces petits lieux de culte en briques rouges, construits à la fin du XIXème siècle sous l'impulsion de l'Impératrice soucieuse de relancer la foi dans ces quartiers ouvriers. En même temps qu'elles offrent un bel exemple de renversement, d'un siècle à l'autre, de leur usage politique, elles n'invitent pas aux macromobilisations que permettent la porte de Brandebourg ou celle de l'Alexanderplatz le 17 juin 1953. Sur la constitution des églises en territoires neutralisés dans un contexte d'affrontement avec la police, voir Siméant J., La cause des sans-papiers, Paris, Presses de ScPo, 1999, p. 322. 
36. . Le quotidien officiel Neues Deutschland titre ainsi le 4 juin 1989 : «L'Armée populaire de libération a écrasé, en Chine, le soulèvement contre-révolutionnaire ", titre dont la qualification des protagonistes ne laisse pas de doute sur ce qu'il convient de penser d'un tel événement. Sur les systèmes d'alarme et de contrôle de l'environnement des acteurs, voir Goffman E., La mise en scène de la vie quotidienne, Paris, Minuit, 1970, t. 2, pp. 241-242, ainsi que Châteauraynaud F., Torny D., Les sombres précurseurs. Une sociologie pragmatique de l'alerte et du risque, Paris, EHESS, 1999, pp. 46-48.

37. . Les prophètes de malheur sont ces « lanceurs d'alarme » qui, « de l'annonce de troubles ou d'accidents inévitables à l'Apocalypse, cherchent à convaincre l'humanité entière qu'une ère de grands malheurs est ouverte » (Châteauraynaud F., Torny D., op. cit., p. 37).

38. . Pour le dire dans les termes de Schelling, le gouvernement peut vouloir grossir la taille réelle des événements protestataires afin de convaincre que, placé dans une sorte de « déficit coercitif », il ne puisse faire autrement qu'employer la force, ce en dépit des dégâts contre lui-même (Stratégie du conflit, Paris, PUF, coll. « Perspectives internationales », 1986 (trad. de l'am. - 1960), pp. 58-61).

39. . Lapp P.-J., « DDR-Kommunalwahlen 1989 », Deutschland Archiv, 22, 1989, pp. 614-617.

40. . Sur la superposition des cycles de mobilisation et des calendriers politiques, voir Fillieule O., Stratégies de la rue, op. cit., 1997, pp. 228-243. Sur la question des cycles, voir la superposition, mi-juillet 1789 , du cycle agricole et de la convocation des Etats généraux (Sewell W., « Historical events as transformation of structures », op. cit., 1996, p. 848).

41. . Une « Wahlparty » (réjouissances post-électorales) fut même organisée dans l'une des églises acquises à la contestation (cf. Rüddenklau W., Störenfried. DDR-Opposition 1986-89, Berlin, 1992 (2ème éd.), p. 291). Mielke avait lui-même décidé que les mesures à prendre devraient être conduites sans être identifiables par les tiers (Au_enstehende). 42. . On retrouve ici l'effet paradoxal de la répression sur le groupe des « déviants politiques » (cf. infra n. 9 et Opp K.-D., « DDR'89 », op. cit., 1993).

43. . Il faudrait ici évoquer les journées de la gare de Dresde, qui se déroulèrent jusque la veille des journées «fatales » (cf. Süß W., Staatssicherheit am Ende, op. cit., 1999, p. 306) de Leipzig pour dépeindre au plus juste l'environnement cognitif des acteurs de Leipzig. Disons simplement que les enseignements tirés de Dresde furent les mêmes des deux côtés : l'humiliation subie à Dresde par le gouvernement ne pouvait pas se répéter, sauf à manifester l'impuissance définitive du régime. Les journées de Dresde avaient exposé aux yeux de tous ce qui ne devait en aucun cas se reproduire ailleurs, et ce qui contribuait alors à faire du lundi 9 à Leipzig le jour J. Ajoutons par ailleurs que c'est à Leipzig que les manifestations les plus dures s'étaient jusqu'alors déroulées (des interpellations se produisirent, nous l'avons dit, dès septembre 1989, d'autres encore le 7 octobre). Voir l'interprétation en ce sens de Süß W., Staatssicherheit am Ende, op. cit., 1999, p. 306 et 314 (voir néanmoins p. 315 n. 69), ainsi que Kuhn E., Der Tag der Entscheidung. Leipzig, 9. Oktober 1989, Berlin, 1992. Ce qui nous importe ici n'est pas de trancher dans cette lutte en vue de la détermination du point décisif ou du jour-J, mais de comprendre comment un événement pèse sur l'autre et en détermine donc la morphologie. Le 9 octobre débute ainsi dans la débandade de Dresde (sur l'interdépendance des faits et de leur interprétation, voir Baker K., Au tribunal de l'opinion, op. cit., 1990). 
44. . La mise en scène de la vie quotidienne, op. cit., 1970, pp. 186-191.

45. . La peur est d'ailleurs l'une des raisons pour lesquelles les acteurs ignoraient parfois leur raison d'être là : puisqu'une réaction politique répressive était à craindre, et puisque les répertoires d'action policiers étaient centrés sur le contrôle de l'accès au lieu de rassemblement, personne n'osait bien entendu pénétrer sur les lieux muni de banderole. Ces manifestations présentent en conséquence l'allure curieuse de groupes compacts agrégés devant le porche d'une église (voir les photographies in Kuhn E., « Wir sind das Volk». Die friedliche Demonstration in Leipzig, 9. Oktober 1989, Berlin, Ullstein, 1999 (2ème éd.).

46. . Cf. infra note 25 .

47. . « Nous sommes prêts (...) à mettre un terme efficace et définitif à ces actions contre-révolutionnaires. Et s'il le faut, les armes à la main » (document rapporté par Neues Forum Leipzig, Jetzt oder nie - Demokratie !, Leipzig, 1989, p. 63).

48. . Sur « scènes de l'action publique » et " scènes officieuses ", voir Corcuff P., Sanier M., « Politique publique et action stratégique en contexte de décentralisation. Aperçus d'un processus décisionnel 'après la bataille' », Annales HSS, 55, (4), 2000, p. 858.

INDEX

Index géographique : Allemagne

Mots-clés : crises politiques, mouvements politiques, police

Index chronologique : 1989 